\title{
Tearing and MHD Instability During Gun Injection into a Spheromak
}

\author{
T.K. Fowler
}

May 11, 2000

U.S. Department of Energy

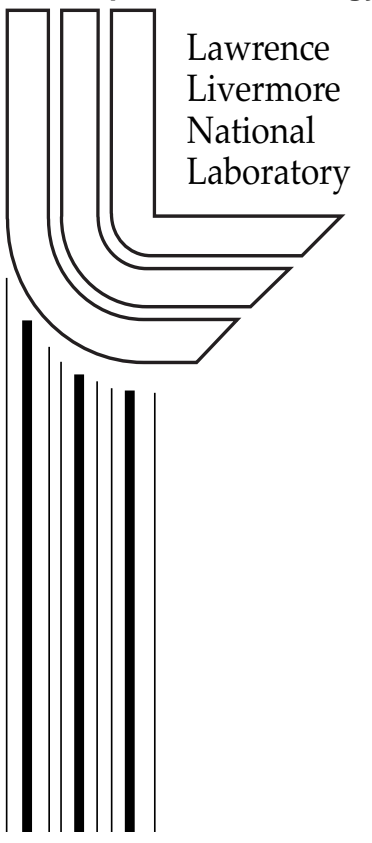




\section{DISCLAIMER}

This document was prepared as an account of work sponsored by an agency of the United States Government. Neither the United States Government nor the University of California nor any of their employees, makes any warranty, express or implied, or assumes any legal liability or responsibility for the accuracy, completeness, or usefulness of any information, apparatus, product, or process disclosed, or represents that its use would not infringe privately owned rights. Reference herein to any specific commercial product, process, or service by trade name, trademark, manufacturer, or otherwise, does not necessarily constitute or imply its endorsement, recommendation, or favoring by the United States Government or the University of California. The views and opinions of authors expressed herein do not necessarily state or reflect those of the United States Government or the University of California, and shall not be used for advertising or product endorsement purposes.

This work was performed under the auspices of the U. S. Department of Energy by the University of California, Lawrence Livermore National Laboratory under Contract No. W-7405-Eng-48.

This report has been reproduced directly from the best available copy.

Available electronically at http://www.doc.gov/bridge

Available for a processing fee to U.S. Department of Energy

And its contractors in paper from

U.S. Department of Energy

Office of Scientific and Technical Information

P.O. Box 62

Oak Ridge, TN 37831-0062

Telephone: (865) 576-8401

Facsimile: (865) 576-5728

E-mail: reports@adonis.osti.gov

Available for the sale to the public from

U.S. Department of Commerce

National Technical Information Service

5285 Port Royal Road

Springfield, VA 22161

Telephone: (800) 553-6847

Facsimile: (703) 605-6900

E-mail: orders@ntis.fedworld.gov

Online ordering: http://www.ntis.gov/ordering.htm

OR

Lawrence Livermore National Laboratory

Technical Information Department's Digital Library

http:/ / www.llnl.gov/tid/Library.html 


\title{
Tearing and MHD Instability During Gun Injection into a Spheromak T. K. Fowler \\ May 11, 2000
}

\begin{abstract}
Linear stability analysis of a two-cylinder approximation to gun injection -- one cylinder to represent the confined spheromak and another to represent the gun -- is shown to yield equilibria in which tearing modes exist simultaneously at the magnetic axis and at the geometric (gun) axis, as might be required to sustain helicity injection. These equilibria are MHD stable at the two axes but may have localized MHD instability at an interior minimum in the q profile. The theory predicts two tearing thresholds with successively deeper q minima as the gun current is increased at constant bias flux.
\end{abstract}

\section{Introduction}

The purpose of this note is to call attention to theoretical results in the literature that shed light on gun injection in SSPX.

We draw upon two points. First, Robinson has published tractable instability criteria for MHD internal kink modes and tearing modes near the axis of a force-free straight cylinder [1]. Secondly, Jarboe has pointed out that a spheromak can be approximated by two straight cylinders, one along the magnetic axis to represent the spheromak core and one along the geometric axis that includes the open-line gun "flux core" and closed surfaces near the flux core [2]. Applying Robinson's criteria separately to each cylinder gives conditions for maintaining MHD stability while allowing tearing instability that may be required to propagate helicity into or out of the respective cylinder.

We do find scenarios meeting these requirements. Since the criteria apply on axis, these scenarios remain valid even as the gun flux core radius shrinks to zero as the spheromak current rises indefinitely at fixed gun current. Thus, we do not find any stability limitation on the ultimate field that can be achieved by sustained helicity injection. Moreover, our scenarios have only $\mathrm{n}=1$ modes at the magnetic axis, as observed in experiments, and the highest lambda ratio allowing resonance at both axes is $\lambda_{\text {gun }} \lambda_{\text {Taylor }} \approx$ 2.5 , similar to the "sweet spot" that maximizes the field obtained by varying the gun current versus bias flux using the Formation Bank. However, we find that injection by tearing requires a minimum in q in the interior, possibly a source of internal MHD instability [3]. 
Also, more work is needed to interpret recent results with the Sustainment Bank, discussed in Section 4.

\section{Stability Criteria}

We describe the equilibrium by:

$$
\lambda=\mu_{\mathrm{o}} \mathbf{j} \cdot \mathbf{B} / \mathrm{B}^{2}=(2 \pi / \mathrm{L})[2 \mathrm{q}-\mathrm{r}(\mathrm{dq} / \mathrm{dr})]\left[\mathrm{q}^{2}+(2 \pi \mathrm{r} / \mathrm{L})^{2}\right]^{-1}
$$

where $r$ is the radial distance in a cylinder and $L$ is its length. We expand $\lambda$ as:

$$
\lambda \quad=\quad \lambda_{1}\left[1+\alpha\left(2 \pi \mathrm{r} / \mathrm{Lq}_{1}\right)^{2}+\cdots\right]
$$

Substituting this into Eq. (1) gives:

$$
\begin{array}{ll}
\lambda_{1}=\left(4 \pi / \mathrm{Lq}_{1}\right) \\
\mathrm{q}=\mathrm{q}_{1}\left[1+\gamma\left(2 \pi \mathrm{r} / \mathrm{Lq}_{1}\right)^{2}+\cdots\right] \\
\gamma=-(\alpha+1) / 2=\left[1 / 2(\mathrm{Lq} / 2 \pi) \mathrm{d}^{2}(\mathrm{Lq} / 2 \pi) / \mathrm{dr}^{2}\right]_{\mathrm{r} \rightarrow 0}
\end{array}
$$

where $\gamma$ is Robinson's stability parameter. As a verification of Jarboes's two cylinder concept, note that applying Eq. (3) with L equal to the circumference at the magnetic axis and $\mathrm{L}$ equal to the diameter at the geometric axis does give the correct $\mathrm{q}$ ratio for the Taylor state in a spherical flux conserver [2].

Robinson's criteria for instability on axis are:

$$
\begin{array}{llll}
\left(\mathrm{m}^{2} / 4\right)+\gamma^{-1}+2< & 0 & \text { MHD } \\
4 \gamma^{-1}>2 \mathrm{~m}-7 & & & \text { Tearing }
\end{array}
$$


where $\mathrm{m}$ is the azimuthal wave number $(\mathrm{m} \neq 0)$. Substituting for $\gamma$ by Eq. (5) gives the stability boundaries plotted in Figure 1:

$$
\begin{array}{ll}
\alpha>-1 \text { and } \alpha<-\mathrm{m}^{2} /\left(8+\mathrm{m}^{2}\right) & \text { MHD } \\
\alpha<-1 \text { and } \alpha>-(1+2 \mathrm{~m}) /(2 \mathrm{~m}-7) & \text { Tearing }
\end{array}
$$

\section{Application to Gun Injection}

During injection, with $\lambda$ decreasing from the geometric axis toward the magnetic axis, the upper half plane in Figure 1 describes the magnetic axis, with $\alpha>0$, while the lower half plane describes the geometric axis, with $\alpha<0$. Thus we see right away that MHD kinking can occur in the gun channel (geometric axis) but not at the magnetic axis. Moreover, tearing and MHD stability are mutually exclusive, so that stimulating tearing in order to transport helicity out of the gun channel also stabilizes MHD modes in the gun channel.

Requiring that tearing instability occur at the geometric axis places a strong constraint, that $\alpha=-(1+2 \varepsilon)<-1$ at this axis, for a small positive $\varepsilon$. Neglecting $\varepsilon$ in $\lambda$, we obtain at the geometric axis:

$$
\begin{aligned}
& \lambda \mathrm{a}=2 \pi \mathrm{q}_{\mathrm{a}}\left(1-\left[\mathrm{q}_{\mathrm{a}} \pi(\mathrm{a}-\mathrm{r}) / \mathrm{a}\right]^{2}+\cdots\right) \\
& \mathrm{q}=\mathrm{q}_{\mathrm{a}}\left(1-\varepsilon\left[\mathrm{q}_{\mathrm{a}} \pi(\mathrm{a}-\mathrm{r}) / \mathrm{a}\right]^{2}+\cdots\right)
\end{aligned}
$$

In deriving these expressions from Eqs. (2) - (4), we take $\mathrm{L}=\mathrm{R}=2 \mathrm{a}$ for SSPX with flux conserver radius $\mathrm{R}$ and spheromak minor radius a (equal to its major radius); and we reserve the symbol $r$ to refer to the magnetic axis and replace $r$ by $(a-r)$ to expand about the geometric axis.

The formula for q in Eq. (11) needs some explanation. In Eq. (4) we define $q=$ $(2 \pi \mathrm{r} / \mathrm{L})\left(\mathrm{B}_{\text {axial }} / \mathrm{B}_{\text {azimuthal }}\right)$ for either axis, this being the appropriate $\mathrm{q}$ determining resonance surfaces and stability in Robinson's criteria. For the two-cylinder model, this cylindrical q 
at the geometric axis is the reciprocal of the usual q relative to the magnetic axis, since $\mathrm{B}_{\text {azimuthal }}$ at the geometric axis is in fact the toroidal field [2]. With an eye toward eventually joining the inner and outer solutions, in Eq. (11) we have adopted the usual definition relative to the magnetic axis, Eq. (11) being the reciprocal of Eq. (4) with value $q_{a}$ at the geometric axis.

Turning to the magnetic axis, setting $\mathrm{L}=2 \pi \mathrm{a}$ and denoting $\mathrm{q}_{1} \equiv \mathrm{q}_{\mathrm{o}}$ at this axis in Eqs. (2) - (4) gives at the magnetic axis:

$$
\begin{aligned}
& \lambda \mathrm{a}=\left(2 / \mathrm{q}_{\mathrm{o}}\right)\left(1+\alpha\left(\mathrm{r} / \mathrm{aq}_{\mathrm{o}}\right)^{2}+\cdots\right) \\
& \mathrm{q}=\mathrm{q}_{\mathrm{o}}\left(1-|\gamma|\left(\mathrm{r} / \mathrm{aq}_{\mathrm{o}}\right)^{2}+\cdots\right)
\end{aligned}
$$

We make the conjecture that successful injection requires tearing all the way to the magnetic axis, and accordingly we will take $\alpha$ to be its value at the boundary for tearing instability at the magnetic axis, by Eq. (9) or the upper half of Figure 1, giving as the only possibilities

the three $m$ values $(1,2,3)$ listed in Table 1 . Also, we replaced $\gamma$ by $-|\gamma|$ since $\gamma$ is negative for these cases. Then q given by joining Eqs. (11) and (13) always has a minimum, possibly causing localized MHD activity near this minimum [3].

\section{Injection Scenarios}

To complete the calculation, we attempt to match the $\lambda$ and q formulas in Eqs.(10) (13) at a transition radius where the cylinder along the geometric axis becomes the better description, taken to be $r=0.75 a$. Since this choice of matching radius is approximate and since the only information contained in the formulas is the slope at the two axes, we cannot expect a perfect fit. As a guide, we impose resonance at each axis, as is required for the validity of Robinson's criteria.

Results are given in Table 1, for the three allowed values of $m$ at the magnetic axis and corresponding $\alpha$ ss at the threshold for tearing. We begin by choosing integer values of the toroidal mode number $\mathrm{n}$ at the magnetic axis, which for a given $\mathrm{m}$ determines $\mathrm{q}_{\mathrm{o}}$ to achieve resonance. We judge success by the reasonableness of the predicted resonance obtained at the geometric axis by matching $\lambda$ 's to obtain $\mathrm{q}_{\mathrm{a}}$ at that axis. Recalling that the 
appropriate $q$ value determining resonance at the geometric axis is the reciprocal of $q_{a}$, we require as the two resonances:

$$
\begin{array}{lll}
\mathrm{q}_{\mathrm{o}}=\mathrm{m} / \mathrm{n} & \text { magnetic axis } \\
\mathrm{q}_{\mathrm{a}}=\mathrm{k} / \mathrm{n} & \text { geometric axis }
\end{array}
$$

where as noted the toroidal $\mathrm{n}$ at the magnetic axis is also the azimuthal mode number at the geometric axis and $\mathrm{k}$ is the axial mode number there.

As it turns out, only certain n's at the magnetic axis allow matching $\lambda$ 's for any value of $\mathrm{q}_{\mathrm{a}}$, resonant or not; and we have not found solutions matching q's for $\mathrm{m}=2$ or 3 . As indicated in Table 1, the only allowed solutions correspond to $\mathrm{n}=1$ at the magnetic axis as observed in experiments, attributable here to the requirement to obtain tearing at the magnetic axis. We find two solutions for $\mathrm{m}=1$, corresponding to different axial $\mathrm{k}$ values at the geometric axis and correspondingly different $q$ values and different values of $\lambda$ at the geometric axis, labeled $\lambda_{\text {gun }}$, given by Eq. (3) with $\mathrm{q}_{1}=\mathrm{q}_{\mathrm{a}}^{-1}$ and $\mathrm{L}=2 \pi \mathrm{a}$ :

$$
\lambda_{\text {gun }}=\quad \mu_{\mathrm{o}} \mathrm{I} / \psi=2 \pi \mathrm{q}_{\mathrm{a}} / \mathrm{a}
$$

with gun current I and bias flux $\psi$.

In Figures 2 and 3 we plot $\lambda$ and $\mathrm{q}$ for the two cases with $\mathrm{m}=1$ at the magnetic axis. The uncertainty of the fit is indicated by a gap at the fitting radius, $r=0.75 \mathrm{a}$.

Two features of these curves are worth noting.

First, as stressed above, requiring tearing at both axes necessarily causes a minimum in q near the joining radius where the character of flux surfaces shifts from a cylinder along the geometric axis to near-circular surfaces around the toroidal magnetic axis. The reason for this is the opposite signs of Robinson's stability parameter $\gamma$ required to obtain tearing at both axes with the "downhill" lambda profile characterizing injection -giving $\gamma<0$ and hence $\alpha>0$ to obtain $\mathrm{d} \lambda / \mathrm{dr}>0$ as seen from the magnetic axis, and $\gamma>0$ and hence $\alpha<-1$ to obtain simultaneously tearing and also $d \lambda / \mathrm{dr}<0$ as seen from the geometric axis. As already noted, this unavoidable minimum in q may cause localized MHD activity on inner flux surfaces. However, as seen in Figure 3, the minimum in q is 
more shallow for the scenario with lower $\lambda_{\text {gun }}$ and lower $\mathrm{q}_{\mathrm{a}}$, enough so that we have not tried to depict it in this figure due to the uncertainty in the small quantity $\varepsilon=-(\alpha+1)$ determining the slope of q near the geometric axis (see Eq. (11)). We will return to this point in Section 4.

The second point concerns the gun lambda. The two solutions found in Table 1 correspond to a high lambda case with $\left(a \lambda_{\text {gun }}\right) \approx 2 \pi$ and a lower case with lambda half this value. The higher value gives $\lambda_{\text {gun }} / \lambda_{\text {Taylor }}=2.5$, reminiscent of the Formation Bank "sweet spot" as noted earlier.

Together, these two features of our solutions suggest that, as in related problems such as buildup of current in accelerators or the initiation of current in tokamaks, helicity injection into a spheromak may proceed through a sequence of at least two resonance thresholds allowing the simultaneous occurrence of tearing at the gun axis and at the magnetic axis that we conjecture to be required for injection. Each threshold is characterized by $n=1$ at the magnetic axis. The current saturates when the gun power is insufficient to push the current higher, either because helicity transport by tearing matches the power -- as conjectured by Taylor [4] -- or because of MHD activity inherent in the q profile required for injection. The "sweet spot" at $2 \pi$ is the highest threshold according to our conjecture, but also the state with the deepest minimum in $\mathrm{q}$ and potentially greatest vulnerability to internal MHD instability [3].

\section{Comments on Sustainment Bank Operation}

The ideas summarized in Section 3 suggest that the apparent saturation of buildup with the Sustainment Bank may be due to a combination of MHD instability and low gun power.

According to the theory, stimulating tearing at the gun axis requires a steep gradient in $\lambda$ (high gun current), the more so at the highest resonance thresholds that appear to have been achieved with the Formation Bank. According to the lower half of Figure 1, applicable to the gun region, failure to achieve the gradient required for tearing induces instead MHD modes at the gun axis at a much lower gradient, with threshold $\alpha=-0.11$ at $\mathrm{n}=1$ (recalling that in the lower half of the graph $\mathrm{m}$ means the toroidal mode number $\mathrm{n}$ at the geometric axis). On the other hand, achieving tearing may cause the onset of MHD on interior surfaces near the open-line gun channel. Either way, a region controlled by MHD may fail to reconnect, leaving a large volume connected to open lines. 
Low available gun power may exaggerate the problem. The pre-existing spheromak created by the Formation Bank already has a large value of $\lambda$ at the magnetic axis, thus making it difficult to achieve the steep profile required for tearing at the geometric axis.

Experiments in which the Sustainment Bank itself creates the spheromak, or in which the pre-exisitng spheromak is made deliberately weaker, might help clarify these issues.

\section{Future Work}

These preliminary results suggest that a more thorough study of linear stability may shed further light on helicity injection, important in its own right and in support of nonlinear calculations on Nimrod.

The easiest improvement to implement would be the application of Robinson's onaxis instability criteria at the magnetic and geometric axes of Corsica calculations, either in terms of our parameter $\alpha$ obtained by a parabolic fit to $\lambda$ as in Eq. (2) or by directly calculating Robinson's stability parameter $\gamma$ form the q profile using Eq. (5) -- always adjusting the $\lambda$ profile to give q's resonant at the two axes as is required for validity of Robinson's criteria.

More stability analysis using the cylinder model could show whether or not MHD and/or tearing modes exist in the interior region, using the equations in the Appendix and Ref. [1], similar to Jardin's work on MHD modes in cylinder approximation but using more realistic q profiles calculated in Corsica [5].

Finally, the cylindrical model can also be used to calculate free energy and bounds on fluctuations, as discussed in the Appendix. 
Appendix: Cylinder Model of MHD and Tearing Modes in Spheromaks

We briefly review the straight cylinder model given in Reference [1], first for the force-free case. Boozer [6] notes that tearing modes occur if there exists a neighboring equilibrium of lower energy when reconnection is allowed, given by:

$$
\nabla \times \mathbf{B}=\quad \lambda(\psi) \mathbf{B}
$$

with equilibrium $\mathbf{B}_{\mathrm{o}}$ and $\lambda_{\mathrm{o}} \equiv \lambda\left(\psi_{\mathrm{o}}\right)$ where $\psi$ describes flux surfaces. We perturb this equation with $\mathbf{b}=\mathbf{B}-\mathbf{B}_{0}$ and $\delta \psi=\psi-\psi_{\mathrm{o}}$, giving:

$$
\begin{array}{lll}
\nabla \times \mathbf{b}= & \lambda_{\mathrm{o}} \mathbf{b}+\quad\left(\mathrm{d} \lambda_{\mathrm{o}} / \mathrm{d} \psi_{\mathrm{o}}\right) \delta \psi \mathbf{B}_{\mathbf{o}} \\
\mathbf{B} \cdot \nabla \psi= & \mathbf{b} \cdot \nabla \psi+\quad \mathbf{B}_{\mathrm{o}} \cdot \nabla \delta \psi=0
\end{array}
$$

where we use $\operatorname{div} \mathbf{B}=0$ in Eq. (A3). Let $\mathbf{b}$ and $\delta \psi$ vary as $\exp \mathbf{i k} \cdot \mathbf{x}$ with $\mathbf{k}=\theta \mathrm{m} / \mathrm{r}+\mathbf{z k}_{\mathrm{z}}$. Using $\mathbf{B}_{0} \cdot \nabla=\mathrm{ik} \cdot \mathbf{B}_{\mathrm{o}}$ gives:

$$
\begin{aligned}
\nabla \times \mathbf{b} & \left.=\lambda_{\mathrm{o}} \mathbf{b}+\left(\mathrm{d} \lambda_{\mathrm{o}} / \mathrm{dr}\right)\right)\left(\mathrm{b}_{\mathrm{r}} / \mathbf{k} \cdot \mathbf{B}_{\mathrm{o}}\right) \mathbf{B}_{\mathrm{o}} \\
\nabla \cdot \mathbf{b} & =0
\end{aligned}
$$

The right side of Eq. (A4) properly satisfies $\nabla \cdot \mathbf{j}=0$.

Combining Eq. (A5) and the $r$ component of Eq. (A2) gives:

$$
\begin{aligned}
& b_{\theta}=\mathrm{i} \mathrm{K}^{-1}\left[\mathrm{md}\left(\mathrm{rb}_{\mathrm{r}}\right) / \mathrm{dr} \quad+\mathrm{k}_{\mathrm{z}} \mathrm{r}^{2} \mathrm{~m} \lambda_{\mathrm{o}} \mathrm{b}_{\mathrm{r}}\right] \\
& b_{z}=i K^{-1}\left[k_{z} r d\left(r_{r}\right) / d r \quad-m \lambda_{o} r_{r}\right]
\end{aligned}
$$


with $K=\left[m^{2}+\left(k_{z} r\right)^{2}\right]$. Finally, introducing Eqs. (A6) and (A7) into either the $\theta$ or $z$ component of Eq. (A2) and dividing by $\mathrm{m}^{2}$ gives the stability equation:

$$
\begin{aligned}
\mathrm{d} / \mathrm{dr}\left(\mathrm{r} \mathrm{K}^{-1} \mathrm{~d}\left(\mathrm{rb}_{\mathrm{r}}\right) / \mathrm{dr}\right) & +\left[\mathrm{K}^{-1} \lambda_{\mathrm{o}}{ }^{2} \mathrm{r}^{2}+2 \mathrm{~K}^{-2} \mathrm{kmr}^{2} \lambda_{\mathrm{o}}-1\right] \mathrm{b}_{\mathrm{r}} \\
& =\mathrm{K}^{-1} \mathrm{r}\left(\mathrm{d} \lambda_{\mathrm{o}} / \mathrm{dr}\right)\left[\left(\mathrm{mB}_{\mathrm{oz}}-\mathrm{k}_{\mathrm{z}} \mathrm{rB}_{\mathrm{o} \theta}\right) / \mathbf{k} \cdot \mathbf{B}_{\mathrm{o}}\right] \mathrm{b}_{\mathrm{r}}
\end{aligned}
$$

and the corresponding energy:

$$
\begin{aligned}
\delta \mathrm{W}=2 \pi \mathrm{L} \int \mathrm{rdr} & \left(2 \mu_{\mathrm{o}}\right)^{-1}\left\{\mathbf{b}^{2}-\left[2 \mathrm{~K}^{-1} \lambda_{\mathrm{o}}{ }^{2} \mathrm{r}^{2}+2 \mathrm{~K}^{-2} \mathrm{kmr}^{2} \lambda_{\mathrm{o}}\right] \mathrm{b}_{\mathrm{r}}{ }^{2}\right. \\
+ & \left.\mathrm{K}^{-1} \mathrm{r}\left(\mathrm{d} \lambda_{\mathrm{o}} / \mathrm{dr}\right)\left[\left(\mathrm{mB}_{\mathrm{oz}}-\mathrm{k}_{\mathrm{z}} \mathrm{rB}_{\mathrm{o} \theta}\right) / \mathbf{k} \cdot \mathbf{B}_{\mathrm{o}}\right] \mathrm{b}_{\mathrm{r}}{ }^{2}\right\}
\end{aligned}
$$

Though somewhat different in appearance, Eq. (A8), which is the Euler equation for variation of $\delta \mathrm{W}$, is identical with the stability equation given in Ref. [1]. Differentiable solutions correspond to MHD modes and solutions with discontinuous derivative at resonant surfaces correspond to the outer solution for tearing modes giving $\Delta^{\prime}$ at $r_{\mathrm{s}} \pm \varepsilon$ :

$$
\Delta^{\prime} \quad=\quad\left[\left(\mathrm{db}_{\mathrm{r}} / \mathrm{dr}\right)_{+\varepsilon}-\left(\mathrm{db}_{\mathrm{r}} / \mathrm{dr}\right)_{-\varepsilon}\right] / \mathrm{b}_{\mathrm{r}}
$$

where $\Delta^{\prime}>0$ gives instability.

This form of the stability equation displays directly the $\mathrm{d} \lambda_{\mathrm{o}} / \mathrm{dr}$ driving term of tearing instability. Boozer takes the large aspect ratio limit $\mathrm{kr} \rightarrow 0$ (so $\mathrm{K} \rightarrow \mathrm{m}^{2}$ ) and he drops the $\lambda_{\mathrm{o}}$ terms on the left side of Eq. (A8) to emphasize the gradient drive [6]. Gibson and Whiteman studied the opposite limit $\left(\mathrm{d} \lambda_{\mathrm{o}} / \mathrm{dr}=0\right)$ for $\mathrm{m}=0$ and $\mathrm{m}=1$ [7].

The equilibrium information is contained in $\lambda_{\mathrm{o}}$ which, for a constant value, is the radial wave number for the equilibrium toroidal field, $B_{o z}=J_{o}\left(\lambda_{0} r\right)$. This describes toroidal equilibria ranging from a tokamak with $\lambda_{\mathrm{o}}<<2.4$ to a spheromak with $\lambda_{\mathrm{o}}=2.4$ (zero 
toroidal field at the edge) to an RFP with $\lambda_{\mathrm{o}}>2.4$, in units of 1/a with plasma radius $\mathrm{r}=\mathrm{a}$ taken here to coincide with a conducting wall.

Eq. (A8) yields finite thresholds in $\lambda_{\mathrm{o}}\left(=3.1\right.$ [7]) or in $\mathrm{d} \lambda_{\mathrm{o}} / \mathrm{dr}[1]$ to obtain tearing instability. Thus, to the extent that the straight-cylinder model is applicable, one must interpret Taylor's success in explaining RFP's as being only approximate below the Gibson-Whiteman threshold $\lambda_{\mathrm{o}}=3.1$, the actual state having non-constant $\lambda_{\mathrm{o}}$, but nonetheless almost the same energy as the Taylor state [2].

References [1] and [6] also include pressure-driven resistive modes. For both spheromaks and RFP's, until the $\lambda$ gradient is steep enough to cause current-driven tearing modes, resistive interchange modes that have no linear threshold in pressure are quickly saturated non-linearly by the finite negative value of $\Delta^{\prime}$ below the tearing threshold, and tearing soon dominates for a positive $\Delta^{\prime}$, giving in either case a maximum island width due to pressure of order $\beta / \Delta{ }^{\prime} r_{s}[8]$. Thus tearing is likely to be dominant over resistive interchange at low $\beta$.

Finally, we note that, given the existence of a stable state, we can calculate the free energy and a bound on fluctuations [9], using $\delta \mathrm{W}$ to calculate the energy for a finite perturbation about the stable state with helicity playing the role of the entropy constraint. To find the free energy in a neighboring symmetric equilibrium relative to a symmetric stable state of the same helicity, we can ignore energy and helicity in the small non-symmetric perturbations that drive the system unstable [9] and drop $b_{r}$ terms in $\delta W$, giving as the free energy :

$$
\delta \mathrm{W}=2 \pi \mathrm{L} \int \mathrm{r} \mathrm{dr}\left(\mathbf{b}^{2} / 2 \mu_{\mathrm{o}}\right)
$$

for a perturbation given by $\mathbf{b}=\mathbf{B}_{\mathrm{oi}}-\mathbf{B}_{\text {of }}$ where $\mathrm{i}$ denotes the initial state and $\mathrm{f}$ the final stable state. This also gives a bound on fluctuations [8]. Since the total helicity is conserved aside from minor resistive losses and since the initial and final symmetric states have the same helicity, it follows that the final fluctuation spectrum contains negligible helicity but a finite energy bounded by Eq. (A11). For a stable state with finite $\mathrm{d} \lambda / \mathrm{dr}$, such as the marginally stable states derived above, this free energy is less than that relative to the Taylor state with the same helicity. 


\section{References}

[1] D. C. Robinson, Nuclear Fusion 18, 939 (1978).

[2] T. R. Jarboe, Plasma Phys. Control. Fusion 36, 945 (1994).

[3] D. C. Robinson, Plasma Physics 13, 439 (1971).

[4] J. B. Taylor, Rev. Mod. Phys. 58, 741 (1986).

[5] S. C. Jardin, Nuclear Fusion 22, 629 (1982).

[6] A. H. Boozer, "Plasma Confinement," Encyclopedia of Physical Science and Technology, Academic Press, 1992, Vol. 13, pp. 1 - 20.

[7] R. D. Gibson and K. J. Whiteman, Plasma Phys. 10, 1101 (1968).

[8] M. Kotschenreuther, R. D. Hazeltine and P. J. Morrison, Phys. Fluids 28, 294 (1985).

[9] T. K. Fowler, "Thermodynamics of Unstable Plasmas," Advances in Plasma Physics, A. Simon and W. Thompson, Editors, Interscience, 1968. 
Table 1. Parameters for the Two Cylinder Model

\begin{tabular}{|c|c|c|c|c|c|c|c|c|c|}
\hline \multicolumn{5}{|c|}{ Magnetic Axis } & \multicolumn{5}{|c|}{ Geometric Axis } \\
\hline$\underline{\mathrm{m}}$ & $\mathrm{n}$ & $\mathrm{q}_{0}$ & $\alpha$ & $\gamma$ & $\mathrm{q}_{\underline{a}-}$ & $\mathrm{q}_{\underline{a}}$ & $-\underline{n}$ & $\underline{\mathrm{k}}$ & $\lambda_{\mathrm{gun}}$ \\
\hline 1 & 1 & 1 & 0.6 & -0.8 & 0.5 & 2 & 2 & 1 & $\pi$ \\
\hline 1 & 1 & 1 & 0.6 & -0.8 & 1 & 1 & 1 & 1 & $2 \pi$ \\
\hline 1 & 2 & & & & NO & $\mathrm{SO}$ & ION & & \\
\hline & . . & & & & No $\lambda$ & $\mathrm{OI}$ & $\mathrm{ION}$ & & \\
\hline & -- & - & --- & ---- & --- & - & -- & - & \\
\hline 2 & 1 & 2 & 1.67 & -1.33 & NO & $\mathrm{O}$ & ION & & \\
\hline 2 & 2 & & & & NO & 50 & ION & & \\
\hline 2 & $\ldots$ & & & & NO & $\mathrm{SO}$ & ION & & \\
\hline & -- & . & --- & ---- & -- & - & -- & 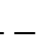 & \\
\hline 3 & 1 & 3 & 7 & -7.5 & NO & $\mathrm{O}$ & ION & & \\
\hline 3 & 2 & & & & $\mathrm{NO}$ & $\mathrm{SO}$ & ION & & \\
\hline 3 & $\ldots$ & & & & NO & 50 & ION & & \\
\hline
\end{tabular}




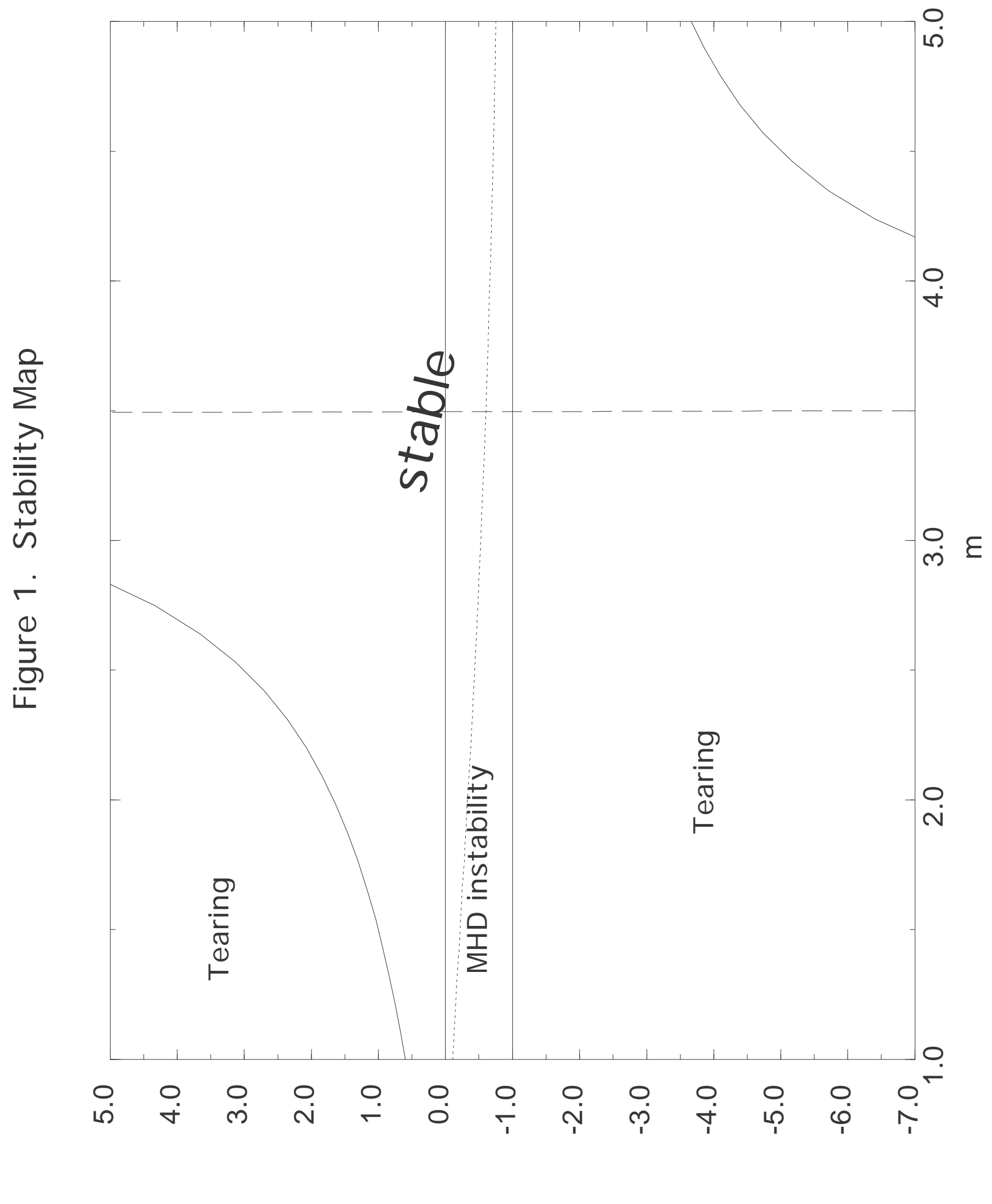




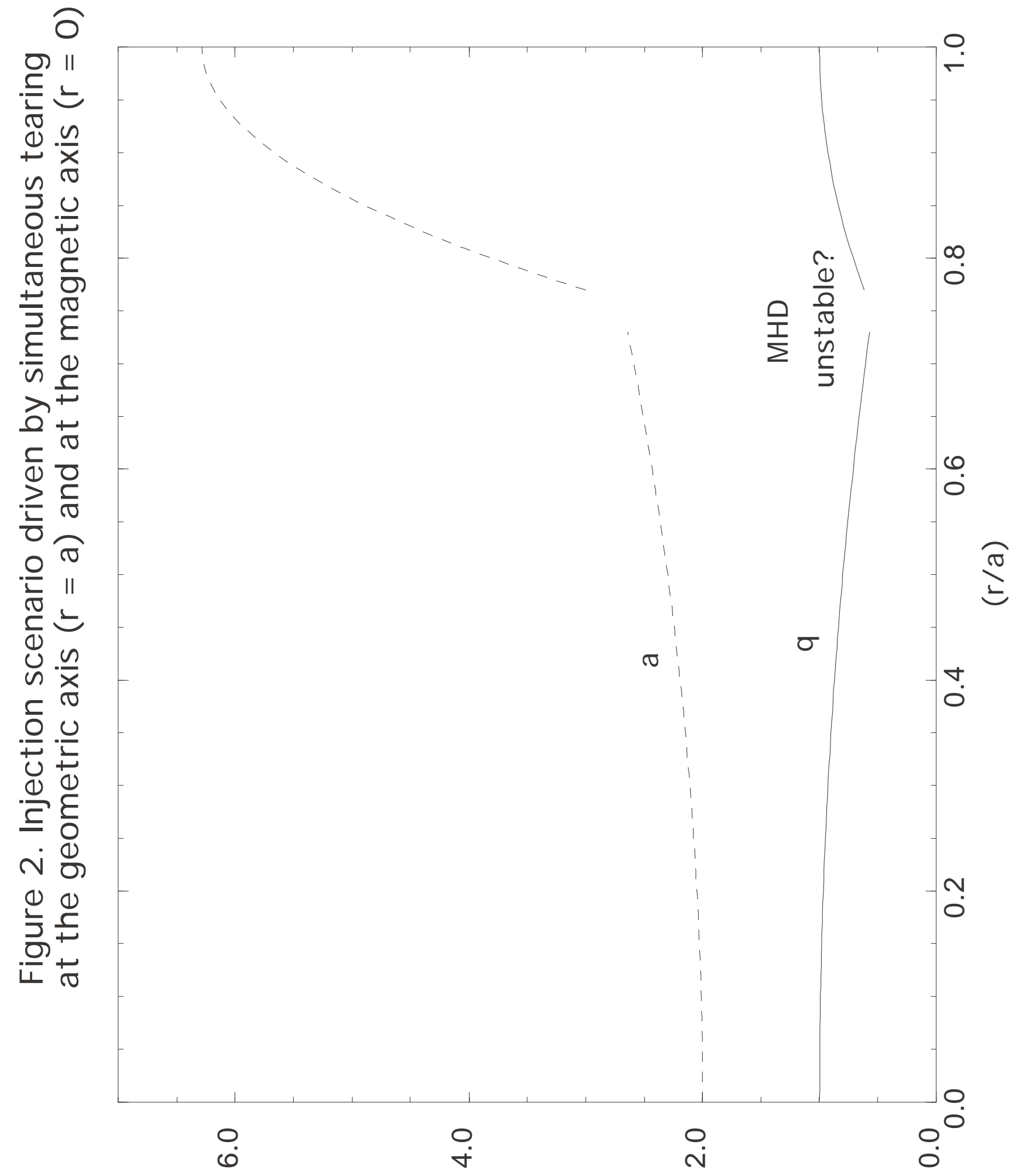




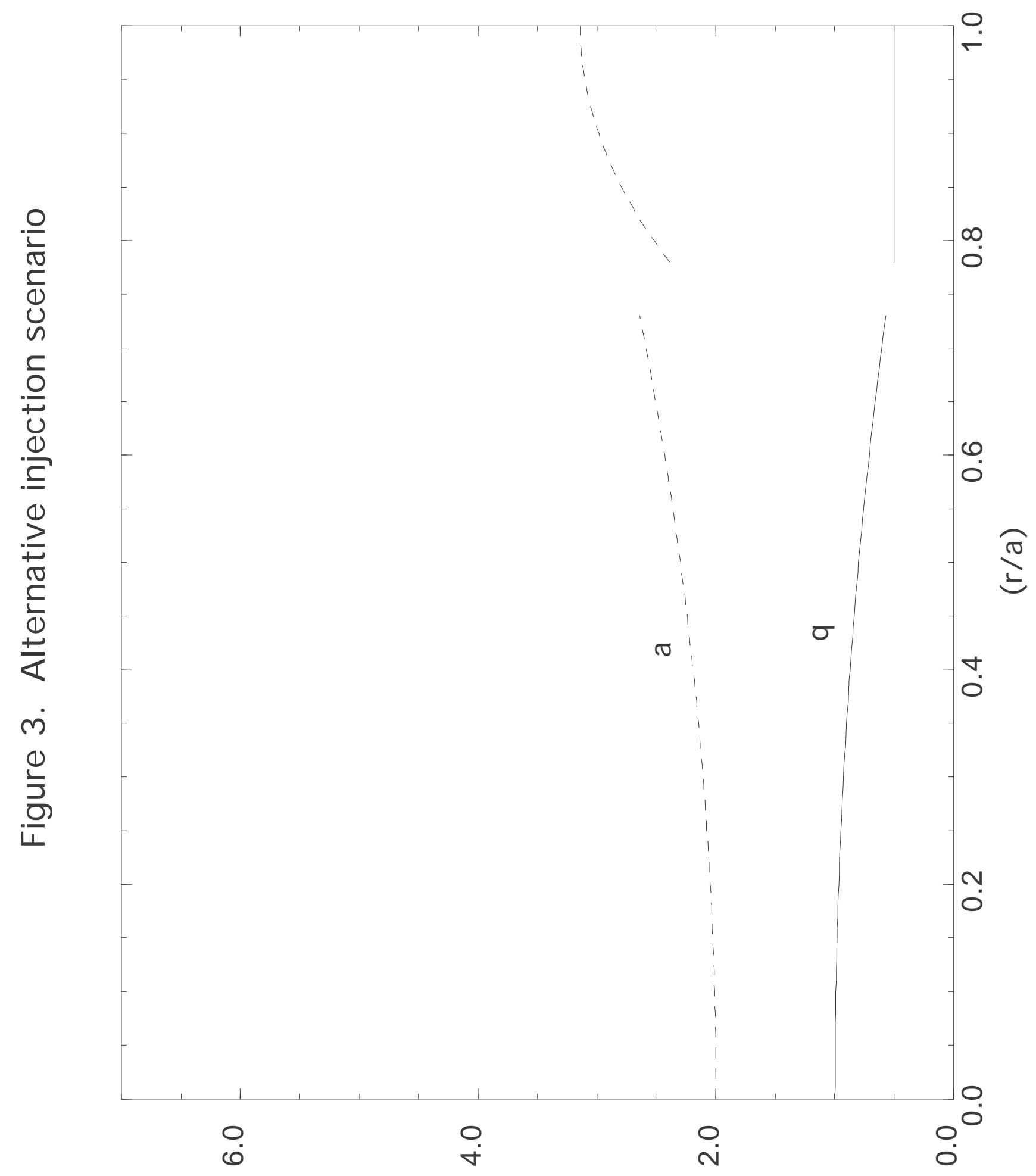

